\title{
Small Quarkonium states in an anisotropic QCD plasma
}

\author{
Yun Gud* \\ Helmholtz Research School, Johann Wolfgang Goethe Universität, \\ Max-von-Laue-Str. 1, D-60438 Frankfurt am Main, Germany \\ Institute of Particle Physics, Huazhong Normal University, Wuhan 430079, China
}

\begin{abstract}
We determine the hard-loop resummed propagator in an anisotropic QCD plasma in general covariant gauges and define a potential between heavy quarks from the Fourier transform of its static limit. We find that the potential exhibits angular dependence and that binding of very small quarkonium states is stronger than in an isotropic plasma.
\end{abstract}

\section{INTRODUCTION}

Information on quarkonium spectral functions at high temperature has started to emerge from lattice-QCD simulations; we refer to ref. [1] for recent work and for links to earlier studies. This has motivated a number of attempts to understand the lattice measurements within non-relativistic potential models including finite temperature effects such as screening [2]. A detailed discussion of the properties of the heavy-quark potential in the deconfined phase of QCD is given in ref. [3], which also provides a comprehensive list of earlier work.

In this paper, we consider the effects due to a local anisotropy of the plasma in momentum space on the heavy-quark potential. Such deviations from perfect isotropy are expected for a real plasma created in high-energy heavy-ion collisions, which undergoes expansion. We derive the HTL propagator of an anisotropic plasma for general covariant gauges, which allows us to define a non-relativistic potential via the Fourier transform of the propagator in the static limit. We also estimate the shift of the binding energy due to the anisotropy.

\section{HARD-THERMAL-LOOP SELF-ENERGY AND PROPAGATOR IN AN ANISOTROPIC PLASMA}

The retarded gauge-field self-energy in the hard-loop approximation is given by [4]

$$
\Pi^{\mu \nu}(p)=g^{2} \int \frac{d^{3} \mathbf{k}}{(2 \pi)^{3}} v^{\mu} \frac{\partial f(\mathbf{k})}{\partial k^{\beta}}\left(g^{\nu \beta}-\frac{v^{\nu} p^{\beta}}{p \cdot v+i \epsilon}\right) .
$$

Here, $v^{\mu} \equiv(1, \mathbf{k} /|\mathbf{k}|)$ is a light-like vector describing the propagation of a plasma particle in space-time. The self-energy is symmetric, $\Pi^{\mu \nu}(p)=\Pi^{\nu \mu}(p)$, and transverse, $p_{\mu} \Pi^{\mu \nu}(p)=0$.

In a suitable tensor basis the components of $\Pi^{\mu \nu}$ can be determined explicitly. For anisotropic systems there are more independent projectors than for the standard equilibrium case [5]. To work in general covariant gauges, we use a four-tensor basis developed in ref. [6] and the self-energy can now be written as $\Pi^{\mu \nu}=\alpha A^{\mu \nu}+\beta B^{\mu \nu}+\gamma C^{\mu \nu}+\delta D^{\mu \nu}$.

In order to determine the four structure functions explicitly we need to specify the phase-space distribution function. We employ the following ansatz:

$$
f(\mathbf{p})=f_{\text {iso }}\left(\sqrt{\mathbf{p}^{2}+\xi(\mathbf{p} \cdot \mathbf{n})^{2}}\right),
$$

*Electronic address: yun@fias.uni-frankfurt.de 
where $\mathbf{n}$ is the direction of anisotropy in momentum space and we choose $\mathbf{n}=(0,0,1)$ in this paper. The parameter $\xi$ is used to determine the degree of anisotropy. Thus, $f(\mathbf{p})$ is obtained from an isotropic distribution $f_{\text {iso }}(|\mathbf{p}|)$ by removing particles with a large momentum component along $\mathbf{n}$.

Since the self-energy tensor is symmetric and transverse, not all of its components are independent. We can therefore restrict our considerations to the spatial part of $\Pi^{\mu \nu}$ and employ the contractions in ref. [6] to determine the four structure functions. We do not list the rather cumbersome explicit expressions for the four structure functions $\alpha, \beta, \gamma$, and $\delta$ here since they have already been determined in ref. [5].

The propagator $i \Delta_{a b}^{\mu \nu}$ is diagonal in color and so color indices will be suppressed. In covariant gauge, its inverse is given by

$$
\begin{aligned}
& \left(\Delta^{-1}\right)^{\mu \nu}(p, \xi)=-p^{2} g^{\mu \nu}+p^{\mu} p^{\nu}-\Pi^{\mu \nu}(p, \xi)-\frac{1}{\lambda} p^{\mu} p^{\nu} \\
& =\left(p^{2}-\alpha\right) A^{\mu \nu}+\left(\omega^{2}-\beta\right) B^{\mu \nu}-\gamma C^{\mu \nu}-\delta D^{\mu \nu}-\frac{1}{\lambda} p^{\mu} p^{\nu}
\end{aligned}
$$

where $\omega \equiv p \cdot m$ and $m^{\nu}$ is the heat-bath vector which equals to $(1,0,0,0)$ in the local rest frame. $\lambda$ is the gauge parameter. Upon inversion, the propagator is written as

$$
\Delta^{\mu \nu}=\frac{1}{p^{2}-\alpha}\left[A^{\mu \nu}-C^{\mu \nu}\right]+\Delta_{G}\left[\left(p^{2}-\alpha-\gamma\right) \frac{\omega^{4}}{p^{4}} B^{\mu \nu}+\left(\omega^{2}-\beta\right) C^{\mu \nu}+\delta \frac{\omega^{2}}{p^{2}} D^{\mu \nu}\right]-\frac{\lambda}{p^{4}} p^{\mu} p^{\nu}
$$

where

$$
\Delta_{G}^{-1}=\left(p^{2}-\alpha-\gamma\right)\left(\omega^{2}-\beta\right)-\delta^{2}\left[\mathbf{p}^{2}-(n \cdot p)^{2}\right]
$$

and the four vector $n^{\mu}=(0, \mathbf{n})$. For $\xi=0$, we recover the isotropic propagator in covariant gauge.

\section{HEAVY QUARK POTENTIAL IN AN ANISOTROPIC PLASMA}

We determine the real part of the heavy-quark potential in the nonrelativistic limit, at leading order, from the Fourier transform of the static gluon propagator,

$$
\begin{aligned}
V(\mathbf{r}, \xi) & =-g^{2} C_{F} \int \frac{d^{3} \mathbf{p}}{(2 \pi)^{3}} e^{i \mathbf{p} \cdot \mathbf{r}} \Delta^{00}(\omega=0, \mathbf{p}, \xi) \\
& =-g^{2} C_{F} \int \frac{d^{3} \mathbf{p}}{(2 \pi)^{3}} e^{i \mathbf{p} \cdot \mathbf{r}} \frac{\mathbf{p}^{2}+m_{\alpha}^{2}+m_{\gamma}^{2}}{\left(\mathbf{p}^{2}+m_{\alpha}^{2}+m_{\gamma}^{2}\right)\left(\mathbf{p}^{2}+m_{\beta}^{2}\right)-m_{\delta}^{4}} .
\end{aligned}
$$

Here, $C_{F}$ is the color factor and the $\xi$-dependent masses $m_{\alpha}^{2}, m_{\beta}^{2}, m_{\gamma}^{2}$ and $m_{\delta}^{2}$ are given in ref. [6].

One may check some limiting cases [6]. The isotropic Debye potential is reproduced if $\xi=0$. On the other hand, when $r \rightarrow 0$ or $\xi \rightarrow \infty$, the potential then coincides with the vacuum Coulomb potential. Generally, the integral in (7) has to be performed numerically. The poles of the function are integrable. They are simple first-order poles which can be evaluated using a principal part prescription. In Fig. 1 we show the potential in the region $\hat{r} \equiv r m_{D} \sim 1$ for various degrees of plasma anisotropy, where $m_{D}$ is the Debye mass. One observes that in general screening is reduced, i.e. that the potential at $\xi>0$ is deeper and closer to the vacuum potential than for an isotropic medium. This is partly caused by the lower density of the anisotropic plasma. However, the effect is not uniform in the polar angle. The angular dependence disappears more rapidly at small $\hat{r}$, while at large $\hat{r}$ there is stronger binding for $\mathbf{r}$ parallel to the direction of anisotropy. Overall, one may therefore expect that quarkonium states whose wave-functions are sensitive to the regime $\hat{r} \sim 1$ are bound more strongly in an anisotropic medium. 

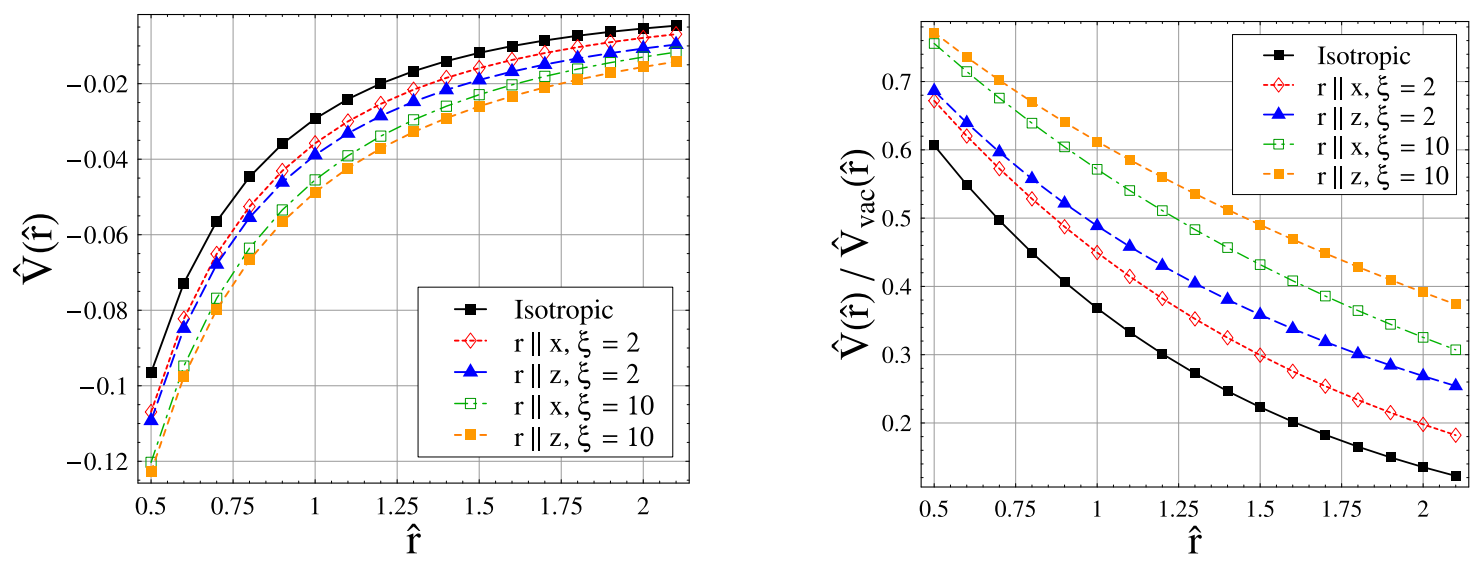

FIG. 1: Heavy-quark potential at leading order as a function of distance $\left(\hat{r} \equiv r m_{D}\right)$ for $\mathbf{r}$ parallel to the direction $\mathbf{n}$ of anisotropy and $\mathbf{r}$ perpendicular to $\mathbf{n}$.

Left: the potential divided by the Debye mass and by the coupling, $\hat{V} \equiv V /\left(g^{2} C_{F} m_{D}\right)$. Right: potential relative to that in vacuum.

\section{BINDING ENERGY FOR SMALL STATES}

Based on the above results, one can determine the binding energy of bound quarkonium states. In particular, we will concentrate on the shift of the binding energy due to the medium. An analytic estimate can be obtained from non-relativistic perturbation theory (to first order) for states with a Bohr radius times Debye mass small as compared to the anisotropy parameter $\xi$.

For weak anisotropy, $\xi \ll 1$, and distances $\hat{r} \ll 1$, we expand the potential as

$$
V(\mathbf{r}) \approx V_{v a c}(r)+\alpha m_{D}-4 \pi \alpha \xi m_{D}^{2} \int \frac{d^{3} \mathbf{p}}{(2 \pi)^{3}} e^{i \mathbf{p} \cdot \mathbf{r}} \frac{\frac{2}{3}-(\mathbf{p} \cdot \mathbf{n})^{2} / \mathbf{p}^{2}}{\left(\mathbf{p}^{2}+m_{D}^{2}\right)^{2}}
$$

where $\alpha \equiv g^{2} C_{F} / 4 \pi$. If $m_{D} / \alpha m_{Q} \ll 1$, where $m_{Q}$ is the quark mass, the vacuum potential dominates and we can use Coulomb wavefunctions to calculate the expectation value of the perturbation given by the last two terms in the equation above. Finally, the binding energy for such small-size states can be expressed as

$$
E_{b i n} \approx E_{v a c}+\alpha m_{D}-\frac{\alpha \xi m_{D}}{6} .
$$

Note that $E_{v a c}<0$. The relative shift of the binding energy due to the presence of the (weakly anisotropic) medium is therefore given by

$$
\frac{\delta E}{E_{v a c}} \approx \frac{4 m_{D}}{\alpha m_{Q}}\left[-1+\frac{\xi}{6}+\cdots\right] \quad, \quad\left(\text { for } \frac{m_{D}}{\alpha m_{Q}} \ll \xi \ll 1\right) .
$$

The first term corresponds to the reduced binding due to screening by the medium while the second term is the correction due to the non-zero anisotropy. The restriction to $m_{D} / \alpha m_{Q} \ll \xi$ is necessary because terms of order $\mathcal{O}\left(m_{D} / \alpha m_{Q}\right)$ inside the brackets have been neglected.

The above analysis applies also to excited states, provided that their size (in units of the Debye length) is small as compared to $\xi$. The shift of the binding energy for the $n^{\text {th }}$ state is

$$
\frac{\delta E}{E_{v a c}} \approx \frac{4 m_{D}}{\alpha m_{Q}} n\left[-1+\frac{\xi}{6}+\cdots\right]
$$


However, it should be clear that for realistic cases (i.e., temperatures, quark masses and coupling constant), our above assumption that $m_{D} / \alpha m_{Q} \ll \xi$ is too extreme. For intermediate-size states and general $\xi$, we must solve exactly the $3 \mathrm{~d}$ Schrödinger equation with the anisotropic potential. This work is in progress.

\section{DISCUSSION AND OUTLOOK}

We have determined the HTL gluon propagator in an anisotropic (viscous) plasma in covariant gauge [6]. Its Fourier transform at vanishing frequency defines a non-relativistic potential for static sources. We find that, generically, screening is weaker than in isotropic media and so the potential is closer to that in vacuum. Also, there is stronger binding of the quark pairs in the anisotropic system. Our results are applicable when the momentum of the exchanged gluon is on the order of the Debye mass $m_{D}$ or higher, i.e. for distances on the order of $\lambda_{D}=1 / m_{D}$ or less.

Following the discussion of the quark potential model in ref. [3], at short distances, there is a string contribution to the potential which is not calculable perturbatively. However, at sufficiently high temperature, the perturbative contribution dominates over the linear confining potential at the length scale $\lambda_{D}$. Roughly, this holds when $T$ is larger than $2 T_{c}$. In this case, our result is directly relevant for quarkonium states with wavefunctions which are sensitive to the length scale $\lambda_{D}$. Conversely, for those states whose length scale is larger, one should sum the medium-dependent contributions due to one-gluon exchange and due to the string [3].

At very short distances, the contribution from the string can also be neglected and one is dealing with perturbed Coulombic states. However, for charmonium or bottomonium, the string contribution is in fact important and it will be interesting to determine their wave functions and binding energies from a potential which is a combination of our anisotropic potential and the string contribution.

\section{Acknowledgments}

The author gratefully acknowledges the collaboration with A. Dumitru and M. Strickland and thanks the Helmholtz foundation, the Otto Stern School at Frankfurt university and Quark-Matter 2008 for financial support.

\section{References}

[1] A. Jakovac, P. Petreczky, K. Petrov and A. Velytsky, Phys. Rev. D 75, 014506 (2007) arXiv:hep-lat/0611017;

G. Aarts, C. Allton, M. B. Oktay, M. Peardon and J. I. Skullerud, Phys. Rev. D 76, 094513 (2007) arXiv:0705.2198 [hep-lat]].

[2] A. Mocsy and P. Petreczky, Phys. Rev. D 73, 074007 (2006) arXiv:hep-ph/0512156.

[3] A. Mocsy and P. Petreczky, Phys. Rev. D 77, 014501 (2008) arXiv:0705.2559 [hep-ph]].

[4] S. Mrowczynski and M. H. Thoma, Phys. Rev. D 62, 036011 (2000) arXiv:hep-ph/0001164.

[5] P. Romatschke and M. Strickland, Phys. Rev. D 68, 036004 (2003) arXiv:hep-ph/0304092.

[6] A. Dumitru, Y. Guo and M. Strickland, Phys. Lett. B 662, 37 (2008) arXiv:0711.4722 [hep$\mathrm{ph}]]$. 